DOI: https://doi.org/10.11144/Javeriana.upsy18-5.cpfe

\title{
Capacidad predictiva de las facetas específicas de Atención plena sobre ansiedad y depresión: Diferencias de Sexo*
}

\section{Predictive capacity of specific Mindfulness facets on Anxiety and Depression: Sex differences}

Recepción: 24 Octubre 2017 | Aceptación: 22 Octubre 2019

María IsABel Jiménez Morales

Universidad Católica San Antonio de Murcia, España ORCID: http://orcid.org/0000-0002-7762-5354

Miguel López-Zamora ${ }^{\mathrm{a}}$

Universidad de Málaga, España

ORCID: http://orcid.org/0000-0003-3466-4465

a Autor de correspondencia. Correo electrónico: miguel.lopez@uma.es

Para citar este artículo: Jiménez Morales, M. I., \& López-Zamora, M. (2019). Capacidad predictiva de las facetas específicas de Atención plena sobre ansiedad y depresión: Diferencias de Sexo. Universitas Psychologica, 18(5), 1-12. https://doi.org/10.11144/Jav eriana.upsy18-5.cpfe

\section{RESUMEN}

La atención plena o mindfulness hace referencia a una disposición general para atender con conciencia el momento presente, que implica la focalización de la misma con una actitud activa de aceptación y libre de juicios. Aunque los beneficios del mindfulness en el ámbito clínico son conocidos, el objetivo de este estudio es analizar las relaciones únicas entre facetas específicas de mindfulness y síntomas concretos de ansiedad y depresión en población española, así como las diferencias de sexo, considerando el efecto de la personalidad. En este estudio se empleó una muestra de 176 sujetos ( $51.7 \%$ mujeres, $48.3 \%$ hombres), con edades comprendidas entre 18 y 69 años $(M=29$; $S D=9.66)$, de las regiones de Murcia y Andalucía (España). Los resultados del análisis de regresión lineal mostraron que, en mujeres con alta ansiedad, la faceta de Describir es capaz de predecir sintomatología ansioso-depresiva, mientras que, en el caso de los hombres, son las facetas de No juzgar y No reaccionar. En mujeres con altas puntuaciones en depresión, las facetas que mejor predicen sintomatología específica son Describir y No juzgar, mientras que, en el caso de los hombres con alta depresión, es la faceta de No juzgar. En conclusión, los resultados podrán contribuir a la mejora y eficacia de las intervenciones basadas en mindfulness para la prevención y tratamiento de problemas de ansiedad y depresión en hombres y mujeres.

Palabras clave

atención plena; mindfulness; ansiedad; depresión diferencias de sexo y personalidad.

\begin{abstract}
The mindfulness refers to a general layout to deal, with awareness, the present moment involving an active and reflective acceptance, and a non-judgmental attitude. Although the benefits of mindfulness in clinical settings are known, the aim of this paper is to analyze the relationships between specific facets of Mindfulness and specific symptoms of anxiety and depression in Spanish population as well as gender differences, considering the personality effects. In this study was used a sample of 176 subjects ( $51.7 \%$ female, $48.3 \%$ male), aged between 18 and 69 years $(M=29 ; S D=9.66)$ residents on Murcia and Andalusia (Spain). The
\end{abstract}


results showed that in women with high anxiety, Describe facet is able to predict anxious-depressive symptoms while in the case of man are the facets of Do not judge and Do not react. In women with high depression scores the facets that best predict specific symptoms are Describe and Do not judge, whereas in the case of men with high depression, is the Do not judge facet. In conclusion, the results, could improve the effectiveness of mindfulnessbased interventions for the prevention and treatment of anxiety and depression disorders, both in men and women. Keywords

mindfulness; anxiety; depression; sex differences and personality.

Entre los trastornos psicológicos de mayor prevalencia en las últimas décadas en España se encuentran los de ansiedad y del estado de ánimo. Según datos de la Encuesta Nacional de Salud, ENSE, realizada por el Gobierno de España en 2017 (España, Ministerio de Sanidad, Consumo y Bienestar Social, 2019), se ha detectado que el $6.7 \%$ de los adultos españoles afirma sufrir ansiedad crónica. Estos datos se diferencian notablemente por sexo, ya que las mujeres presentan un porcentaje de 9.1 $\%$, mientras que los hombres alcanzan un $4.3 \%$. Estos porcentajes se repiten con respecto a los problemas relacionados con la depresión, con 6.7 $\%$ de prevalencia para la muestra global y, de nuevo, con el doble de afectación en mujeres $(9.2$ $\%)$ que en hombres (4\%). Se observa, además, un alto riesgo de sufrir estos trastornos $(13.16 \%)$ en población infantil y adolescente, hecho que debe hacernos reflexionar sobre la necesidad de continuar investigando técnicas de intervención eficaces para los trastornos emocionales, así como estrategias orientadas hacia la prevención. En lo que respecta al tratamiento e intervención de los trastornos emocionales, la terapia que cuenta con mayor apoyo empírico es la cognitivo-conductual (TCC) (Lang, 2013), pero recientemente se ha incrementado, de forma notable, el interés por el desarrollo de enfoques alternativos complementarios, como el mindfulness para dichos problemas de salud, que se estima que afectan a más de 350 millones de personas en el mundo (Greeson et al., 2015).

El mindfulness o atención plena, que encuentra su origen en tradiciones orientales adscritas al Budismo Zen, hace referencia a una disposición general para focalizar la conciencia en el momento presente y los fenómenos que se experimentan (pensamientos, emociones, sensaciones, etc.), con una actitud de compasión, interés, apertura y amabilidad (Kabat- Zinn, 2003) y libre de juicios (Brown \& Ryan, 2003; Justo, de la Fuente, \& Salvador, 2011). Desde sus comienzos con los trabajos de Kabat-Zinn en 1970, es considerado un componente central desde diversos enfoques terapéuticos, por lo que muchos profesionales consideran que el mindfulness es un excelente complemento a las terapias clásicas, y en los últimos años se han desarrollado protocolos que la incorporan (Lang, 2013). No obstante, como señalan Jankowski y Holas (2014), la práctica de mindfulness significa cultivar una actitud consciente hacia todo tipo de experiencias y procesos mentales (Smidt \& Vinet, 2015).

Recientemente, el mindfulness se ha conceptualizado a partir de cinco factores o facetas (Baer, Smith, Hopkins, Krietemeyer, \& Toney, 2006; Desrosiers, Klemanski, \& Nolen-Hoeksema, 2013), que son Observar, Describir, Actuar con conciencia, No juzgar y No reaccionar.

El mindfulness es un constructo multifacético que, como podemos observar en la literatura psicológica actual, se ha descrito como un estado un estado, un rasgo (Brown \& Ryan, 2003), y también como un proceso que implica la autorregulación de la atención (Bishop et al., 2004). De este modo, el mindfulness, entendido como un estado, implicaría la consciencia que emerge al prestar atención de forma deliberada en el momento presente con una actitud curiosa, abierta y de aceptación hacia el objeto de observación. La práctica habitual de estados de atención plena (ya sea formal o informal) tiende a desarrollar en las personas una serie de capacidades relativamente estables, que es lo que se suele denominar "atención plena rasgo” (Hervás, Cebolla, \& Soler, 2016).

Los modelos terapéuticos actuales basados en la aceptación incorporan la atención plena al tratamiento de la ansiedad con el objetivo de expandir la toma de conciencia del paciente de 
sus respuestas emocionales, que evolucionan de una actitud mental enjuiciadora y controladora hacia una actitud compasiva, lo que enfatiza la flexibilidad cognitiva y la implicación total en el momento presente (Alonso, 2013). Muchos de ellos usan variantes del programa de reducción del estrés basado en mindfulness de Kabat-Zinn, como el entrenamiento en la Terapia cognitiva basada en Atención plena (TCAP) que, como señala Cebolla (2013), se basa en la utilización intencionada del control de la atención para establecer una configuración del procesamiento de la información incompatible con el engranaje depresivo.

En la actualidad existe una gran cantidad de evidencias científicas a favor de los efectos beneficiosos de las intervenciones basadas en el mindfulness para reducir el sufrimiento y promover bienestar, la satisfacción vital y la salud mental, tanto en poblaciones clínicas como en no clínicas (Cuevas-Toro, Díaz-Batanero, DelgadoRico, \& Vélez-Toral, 2017; Hervás et al., 2016; Kabat-Zinn, 2003; Petrocchi \& Ottaviani, 2016). Estas intervenciones en población adulta producen más emociones positivas y menos afecto negativo (ansiedad-depresión), cuando se comparan con grupos de control (Hassed, de Lisle, Sullivan, \& Pier, 2009), reduciendo los síntomas de depresión (Metcalf, Gold, Davis, Sylvia, \& Battle, 2019), así como los sesgos cognitivos que desempeñan un papel central en la etiología y mantenimiento de estos problemas.

Esto ha llevado a un interés creciente por la aplicación de este enfoque en jóvenes y adolescentes (Amutio-Kareaga, Franco Justo, Gázquez Linares, \& Mañas, 2015; Mathews \& MacLeod, 2005; Wazczuk et al., 2015). De hecho, en estudios recientes, señalan la importancia de explorar si existen diferencias en la forma en que los adolescentes de ambos sexos se relacionan con una intervención de mindfulness (por ejemplo, Bluth, Roberson, \& Girdler, 2017). Otra línea de investigación, que es todavía relativamente escasa, tiene que ver con las diferencias de sexo en cuanto a los niveles de mindfulness. Estudios previos corroboran las diferencias de sexo en relación con las competencias emocionales y las estrategias de afrontamiento y regulación emocional, tanto en adultos como en adolescentes (Pascual, Conejero, \& Etxebarria, 2016).

Del mismo modo, la literatura existente indica que la disposición al mindfulness se asocia con un mayor control atencional, una mayor autorregulación emocional de las experiencias emocionales y cambios en la autoconciencia (Hervás et al., 2016). Su relación con el bienestar podría deberse a una mayor reevaluación positiva de los acontecimientos estresantes cotidianos, bajos niveles de rumiación y baja supresión emocional (Dixon \& Overal, 2016). Además, según Ciesla, Reilly, Dickson, Emanuel y Updegraff (2012), parece que es la disposición a no juzgar las experiencias la faceta que disminuye en mayor medida el impacto de los estresores diarios sobre la tristeza. Como señalan estos autores, es posible que el mindfulness reduzca las emociones negativas asociadas a los estresores diarios (Dixon \& Overal, 2016). De este modo, el mindfulness no solo amortigua el impacto de las emociones negativas, sino que además favorece emociones positivas (Hervás et al., 2016), tanto en población general como en poblaciones clínicas, promoviendo el afecto positivo (Zeng \& $\mathrm{Gu}, 2017)$.

Para el tratamiento de la ansiedad y la depresión mediante mindfulness se han encontrado resultados significativos, pero inconsistentes. Algunos estudios de metaanálisis han evidenciado una efectividad diferencial de la intervención basada en mindfulness (MBI), proporcionando un beneficio significativo en la reducción de la severidad de los síntomas depresivos (Greeson et al., 2015), pero no de los síntomas de ansiedad (Strauss, Cavanag, Oliver, \& Pettman, 2014). Otros metaanálisis recientes concluyen que la MBI reduce consistentemente síntomas de estrés, ansiedad y depresión (Hoffman, Sawyer, \& Witt, 2010; Khoury et al., 2013; Goyal et al., 2014). Además, algunas investigaciones sostienen que determinadas características, tales como la severidad de los síntomas de depresión previos a la intervención, actúan como variables moderadoras del efecto beneficioso del entrenamiento en mindfulness (Feagans, Dariotis, Mendelson, \& Greenberg, 
2012), donde no se encontraron hipótesis específicas ni literatura relevante sobre el papel del género o la edad (Greeson et al., 2015). Respecto a los trastornos de ansiedad, la evidencia es más difusa, ya que existen menos estudios de revisión y metanalísticos. Desrosiers et al. (2013) encontraron que la faceta Describir se relaciona negativa y significativamente con activación ansiosa, mientras que Observar lo hace en sentido positivo.

Por ejemplo, Raphiphatthana, Jose y Kielpikowski (2016) revelan en su trabajo que Actuar con conciencia fue la única faceta de atención que predijo significativamente niveles bajos de anhedonia a largo plazo, y que bajas puntuaciones en No juzgar y No reaccionar podían predecir el afecto negativo un mes más tarde. No juzgar y No reaccionar se relacionaron negativamente con depresión y sintomatología depresiva. Por otro lado, la rumiación es uno de los factores clave en la depresión (Manee \& Majreh, 2016), asociada con estados prolongados y severos, y se piensa que podría estar relacionada con algunas facetas de mindfulness. Petrocchi y Ottaviani (2016) han encontrado que la rumiación es capaz de mediar la relación existente entre la faceta de No juzgar y la aparición de síntomas depresivos, ya que los individuos con una alta predisposición al mindfulness suelen tener bajas puntuaciones en rumiación.

Aunque en investigaciones previas (Baer et al., 2006) se ha asociado la práctica del mindfulness con la receptividad a los sentimientos y el interés por los estímulos ambientales y con la reducción del afecto negativo (Smidt \& Vinet, 2015), es probable que las Intervenciones Basadas en Mindfulness (IBM) no sean igualmente eficaces para todos los perfiles, incluso dentro de un mismo trastorno (Wharton \& Kanas, 2019), ya que debemos considerar el papel moderador de la personalidad. Algunos estudios realizados en población no clínica indican que el perfil de personalidad que más puede beneficiarse de las IBM es aquel que incluye puntuaciones elevadas en Neuroticismo y en Responsabilidad (Hervás et al., 2016), aunque apenas existe investigación al respecto.
Tras lo expuesto anteriormente, el objetivo general de este trabajo se articulará en estudiar cómo se relaciona la disposición al mindfulness con rasgos de ansiedad y depresión, así como su relación con factores de personalidad.

El primer objetivo específico de esta investigación será profundizar en la contribución de las facetas específicas de mindfulness a la sintomatología ansioso-depresiva, así como su relación con factores de personalidad en una muestra de población no clínica. Se establecerán dos hipótesis: 1) se producirán relaciones negativas y estadísticamente significativas entre síntomas de ansiedad, depresión, irritabilidad, inutilidad y problemas de pensamiento (rumiación) y las facetas de mindfulness; 2) se producirán relaciones positivas y estadísticamente significativas entre los factores de personalidad y mindfulness, a excepción del factor Neuroticismo, que lo hará en sentido negativo.

El segundo objetivo de nuestro trabajo consistirá en estudiar la existencia de posibles diferencias de sexo en la contribución de las facetas específicas de mindfulness a la aparición de sintomatología ansioso-depresiva, todo ello en población normal. Los objetivos asociados a esta hipótesis serán: 3) encontrar diferencias de sexo en ansiedad. En concreto, planteamos que la faceta de Describir se relacionará con alta ansiedad en las mujeres, mientras que No juzgar predecirá la alta ansiedad en hombres; 4) encontrar diferencias de sexo en depresión. No juzgar será la faceta que predecirá síntomas de depresión, tanto en hombres como en mujeres; y 5) encontrar diferencias de sexo en mindfulness en función de alto o bajo Neuroticismo.

\section{Método}

\section{Participantes y procedimiento}

En este estudio participaron 176 sujetos (51.7 $\%$ mujeres, $48.3 \%$ hombres), con edades comprendidas entre 18 y 69 años $(M=29$; $S D=9.66)$, de las regiones de Murcia y de Andalucía (España). Los participantes aportaron 
su consentimiento informado, informaron que no sufrían ninguna patología clínica susceptible de alterar el estudio, y aceptaron recibir los resultados generales una vez finalizado el mismo. Este estudio cumple todos los principios éticos universales que deben regir la investigación en Psicología.

\section{Instrumentos}

\section{Atención plena}

Para valorar la disposición al mindfulness, los participantes de la muestra cumplimentaron la adaptación Española del Cuestionario de cinco Facetas de Atención Plena (FFMQ, Cebolla et al., 2012), que es una medida de autoinforme, compuesta por 39 ítems que se caracterizan por ser de fácil lectura y comprensión, y cuenta con buenos índices de fiabilidad y validez. La escala Observar evalúa la atención a las experiencias internas y externas, tales como sensaciones, o sentimientos $(\alpha$ para la escala total $=0.81)$. La escala Describir evalúa la capacidad de etiquetar con palabras la experiencia interna $(\alpha=0.91)$. La escala Actuar con conciencia evalúa la capacidad de centrarse en la actividad que se lleva a cabo en el momento $(\alpha=0.89)$. La faceta No juzgar evalúa la actitud no evaluativa del sujeto hacia sus pensamientos y sentimientos $(\alpha=$ 0.91). La escala No reaccionar evalúa en qué medida el sujeto permite que los pensamientos y sentimientos discurran sin verse atrapado por ellos $(\alpha=0.8)$.

\section{Ansiedad-depresión}

Para valorar los síntomas de ansiedad y depresión se empleó el Cuestionario Educativo-Clínico de Ansiedad y Depresión (Lozano, García-Cueto, \& Lozano, 2007), que ofrece una medida de autoinforme dirigida a evaluar los trastornos de la emoción o también denominados trastornos internalizantes, como la depresión y la ansiedad. Está compuesto por 50 ítems de fácil lectura y comprensión, y cuenta con buenos índices de fiabilidad y validez, $(\alpha=0.91)$. La escala Depresión evalúa la presencia de síntomas depresivos $(\alpha=0.8)$. La escala Ansiedad evalúa la presencia de ansiedad $(\alpha=0.7)$. La escala Inutilidad evalúa la creencia del sujeto de su capacidad $(\alpha=0.8)$. La escala Irritabilidad evalúa la consciencia de enfadarse con facilidad $(\alpha$ $=0.81)$. La escala Problemas de pensamiento evalúa la presencia de intrusiones y rumiación $(\alpha$ $=0.7$ ).

\section{Personalidad}

Para la evaluación de la personalidad se ha empleado el Inventario de Evaluación de la Personalidad normal NEO PI-R abreviado (Costa \& McCrae, citados en Sanz, Silvia, \& Avia, 1999). Esta prueba, con un uso muy extendido en la práctica clínica (Aluja, Blanch, Solé, Dolcet, $\&$ Gallart, 2008), presenta una alta fiabilidad y validez $(\alpha=0.87)$. El NEO FFI evalúa 5 factores de personalidad: Neuroticismo $(\alpha=0.9)$, que contrapone el ajuste y la estabilidad emocional al desajuste emocional. Extraversión $(\alpha=0.84)$, que mide la sociabilidad o preferencia por estar rodeado de gente, la vinculación a los grupos, y asertividad. Apertura $(\alpha=0.82)$, que se refiere a la integración activa, atención a los sentimientos interiores, preferencia por la variedad, y la independencia de juicio. Amabilidad $(\alpha=$ 0.83), que mide las tendencias interpersonales, altruistas, empáticas, de disposición a prestar ayuda. Por último, Responsabilidad $(\alpha=0.88)$, la capacidad de planificación y ejecución de las tareas, y que se asocia a un buen rendimiento académico o profesional.

\section{Resultados}

El primer objetivo que se planteó este trabajo fue el de estudiar cómo se relacionan facetas específicas de mindfulness con rasgos de ansiedad y depresión, y con factores de la personalidad. Para ello se realizaron, por separado, dos análisis de correlaciones entre el FFMQ y el Cecad, y entre el FFMQ con el NEO FFI. Antes de llevar a cabo las correlaciones se realizaron dos estudios 
de la varianza, con el objetivo de para comprobar, en primer lugar, que no hubiese diferencias entre sexo en los factores seleccionados para el estudio y, en segundo lugar, si se cumplía el supuesto de homogeneidad, para que los datos no reportaran diferencias poblacionales entre sí. Todos los análisis fueron no significativos $(p>$ 0.05), es decir, que los factores estudiados no mostraron diferencias entre sexo, a excepción de la variable del NEO FFI Apertura $(p=0.034)$, en la que se mostraron unas medias y desviaciones típicas en hombres $(M=32.9 ; S D=7.35)$ ligeramente superiores a las mujeres $(M=30.6$; $S D=7.28)$.

Para comprobar la primera hipótesis de este estudio, se compararon las escalas del FFMQ y del Cecad mediante un análisis de correlación de Pearson para observar si se producían relaciones negativas y estadísticamente significativas entre síntomas de Depresión, Ansiedad, Inutilidad, Irritabilidad y Problemas de Pensamiento (Rumiación, a partir de ahora), y las variables de Observar, Describir, Actuar con conciencia, No juzgar y No reaccionar. Los resultados mostraron que la primera hipótesis se cumplió; todas las variables del Cecad correlacionaron negativamente con las del FFMQ excepto Observar, que correlacionó positivamente con Ansiedad y, en menor medida con Irritabilidad y Rumiación (Tabla 1).

\section{Tabla 1}

Correlaciones entre subescalas del FFMQ con las del Cecad y del NEO FFI

\begin{tabular}{|c|c|c|c|c|c|}
\hline \multirow[b]{2}{*}{ Escalas } & \multicolumn{5}{|c|}{ FFMQ } \\
\hline & Observar & Describir & $\begin{array}{l}\text { Actuar con } \\
\text { conciencia }\end{array}$ & No juzgar & $\begin{array}{c}\text { No } \\
\text { reaccionar }\end{array}$ \\
\hline \multicolumn{6}{|l|}{ Cecad } \\
\hline Depresión & 0.109 & $-0.367(* *)$ & $-0.424(* *)$ & $-0.528(* *)$ & $-0.243(* *)$ \\
\hline Ansiedad & $0.243(* *)$ & $-0.302(* *)$ & $-0.391(* *)$ & $-0.5(* *)$ & -0.137 \\
\hline Inutilidad & 0.021 & $-0.45(* *)$ & $-0.389(* *)$ & $-0.445(* *)$ & $-0.277(* *)$ \\
\hline Irritabilidad & $\left.0.1511^{*}\right)$ & $-0.33(* *)$ & $-0.364(* *)$ & $-0.503(* *)$ & $-0.245(* *)$ \\
\hline Rumiación & $0.179\left(^{*}\right)$ & $-0.366\left(^{(* *)}\right.$ & $-0.429(* *)$ & $-0.59{ }^{(* *)}$ & $-0.27(* *)$ \\
\hline \multicolumn{6}{|l|}{ NEO-FII } \\
\hline Neuroticismo & 0.067 & $-0.34(* *)$ & $-0.4(* *)$ & $-0.507(* *)$ & $-0.417(* *)$ \\
\hline Extraversión & $0.233(* *)$ & $0.229(* *)$ & 0.008 & 0.105 & 0.135 \\
\hline Apertura & $0.383(* *)$ & $0.362(* *)$ & 0.026 & 0.06 & $0.323(* *)$ \\
\hline Amabilidad & 0.123 & 0.116 & 0.095 & $0.151\left(^{*}\right)$ & $0.159\left(^{*}\right)$ \\
\hline Responsabilidad & $0.156\left(^{*}\right)$ & $0.208(* *)$ & $0.613(* *)$ & 0.144 & $0.17(*)$ \\
\hline
\end{tabular}

Para comprobar la segunda hipótesis, se realizó un análisis de correlación de Pearson entre las subescalas del NEO FFI y del FFMQ. Los resultados mostraron correlaciones positivas y estadísticamente significativas entre los factores de personalidad y las facetas de atención plena, a excepción del factor Neuroticismo, que se correlacionó en sentido negativo. Los factores de personalidad Apertura a la experiencia y Responsabilidad fueron los que correlacionaron en mayor medida con las facetas de mindfulness (Tabla 1). Los resultados muestran que la segunda hipótesis se cumplió parcialmente.

El segundo objetivo del presente trabajo fue analizar las diferencias de sexo en la contribución de las facetas específicas de mindfulness a la sintomatología ansioso-depresiva. En primer lugar, se procedió a hacer análisis de regresión múltiple con el método de pasos sucesivos de las variables que corresponden a las hipótesis 3 y 4 del presente trabajo, esto es, Ansiedad y Depresión. Para ello, se dividió la muestra por sexo y se llevaron a cabo por separado análisis de regresión lineal múltiple con pasos sucesivos, estableciendo como variables dependientes las dimensiones del Cecad y del NEO FFI, y como variables independientes y predictores, las subescalas de la batería FFMQ. Adicionalmente, la muestra de sexos fue segmentada mediante un proceso habitual en el estudio de diferencias individuales en tres grupos según la variable en cuestión, y se tomaron únicamente los grupos con alta ansiedad y alta depresión. Esto permitió estudiar cómo se comportan los sexos cuando sus puntaciones se encuentran maximizadas. Para mayor claridad, se reportan únicamente los modelos con mejores resultados.

\section{Análisis de ansiedad}

En el análisis de los resultados del grupo de mujeres de la muestra, se encontraron diferencias cuando se compararon en función de su nivel de ansiedad. En las mujeres con alta ansiedad, la faceta Describir fue capaz de predecir todas las subescalas del Cecad. Por el contrario, en la muestra de hombres, fueron las facetas de No Juzgar y No reaccionar las que predijeron mejor el Cecad (Tabla 2). 


\section{Tabla 2}

Regresiones lineales múltiples entre subescalas del FFMQ y alta Ansiedad (Cecad) en mujeres y hombres

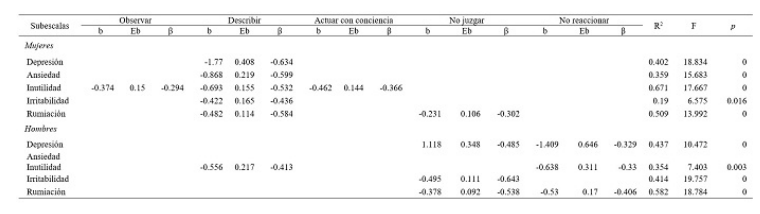

Análisis de depresión

En el análisis de los resultados del grupo de mujeres se apreciaron también diferencias cuando se compararon en función de sus puntuaciones en depresión. Las facetas que mejor predijeron subescalas del Cecad en mujeres con alta depresión fueron las de describir y no juzgar. Por el contrario, en hombres con alta depresión fue la faceta No juzgar la más predictora (Tabla 3).

\section{Tabla 3}

Regresiones lineales múltiples entre subescalas del FFMQ y alta Depresión (Cecad) en mujeres

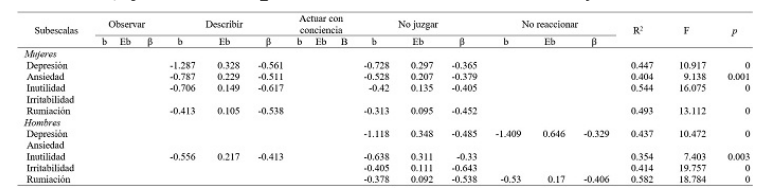

Para comprobar la hipótesis 5 de este trabajo, por la que se esperaba encontrar diferencias de género en mindfulness en función de alto o bajo Neuroticismo, se procedió a dividir a la muestra según su sexo y sus puntuaciones en dicha variable, y después se llevaron a cabo regresiones lineales múltiples con las facetas del FFMQ como variables independientes. No se encontró relación entre las variables de mindfulness y bajo neuroticismo (todas las $\mathrm{p}>0.05$ ). Sin embargo, cuando se llevaron a cabo las regresiones con alto neuroticismo, sí que aparecieron datos de interés. Las facetas de Observar y No juzgar predicen un alto neuroticismo, pero con una diferencia, mientras que en mujeres la relación es negativa (a mayores puntuaciones en Observar y No juzgar, menos Neuroticismo), en hombres se produce al contrario (Tabla 4).
Tabla 4

Regresiones lineales múltiples entre subescalas del FFMQ y Neuroticismo (NEO FFI) en mujeres y hombres

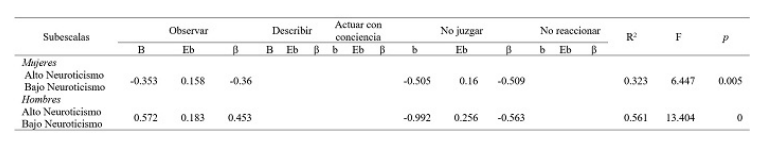

\section{Discusión}

El primer objetivo de este estudio ha sido profundizar en la contribución de las facetas específicas de mindfulness a la sintomatología ansioso-depresiva en una muestra de población no clínica, así como su relación con factores de personalidad.

En la primera hipótesis se planteaba que se producirían relaciones negativas y estadísticamente significativas entre síntomas de ansiedad-depresión y las facetas de mindfulness. Los resultados obtenidos, en la misma línea de otros estudios recientes (Ciesla et al., 2012), confirmaron que, cuanto mayor es la capacidad de centrarse en la actividad sin juzgar o tener una actitud evaluativa hacia los pensamientos y sentimientos y a etiquetarlos, menos síntomas de depresión, ansiedad, inutilidad, irritabilidad y rumiación. Una posible explicación es que las personas con un comportamiento en "piloto automático," que presenta una disposición atencional dirigida hacia el futuro o el pasado y preocupación por los pensamientos fantasías y planes, presentan una mayor tendencia a experimentar afecto negativo, donde ese estado de ausencia de mindfullnes (o mindlessness) es el que obstaculiza el reappraisal cognitivo, y que la persona pueda realizar interpretaciones más positivas de las experiencias estresantes, lo que genera a su vez más síntomas de ansiedad y depresión. Estos resultados van en la línea de otros recientes (Geiger et al., 2016; Zeng \& Gu, 2017).

Con respecto a la segunda hipótesis del estudio, los datos confirmaron que la disposición al mindfulness se relaciona negativamente con el factor Neuroticismo, y positivamente con el resto de los factores de la personalidad. 
En concreto, se observa que la inestabilidad emocional que caracteriza a las personas muy neuróticas se relaciona con una alta tendencia a juzgar y una alta reactividad ante la experiencia, así como la incapacidad para centrarse en el momento presente sin etiquetarlo. Posiblemente las personas neuróticas, con altos niveles de autoexigencia y alta necesidad de control, tienen a realizar más juicios y más negativos sobre sí mismos y sobre sus pensamientos y experiencias pasadas y futuras. Una posible explicación es que la ausencia de mindfulness que les caracteriza desencadena procesos de carácter rumiativo y la aparición de afecto negativo. Estudios recientes evidencian que el Neuroticismo se relaciona con la depresión y el importante el papel mediador que ejercen los niveles de rumiación y de tendencia a la preocupación (Manee \& Majreh, 2016).

El estado de mindlessness, en el que quedan inmersas las personas neuróticas que pretenden aumentar su sensación de control y autoeficacia percibida, supone anticiparse o enjuiciar acontecimientos sobre los cuales la persona ha tenido o tiene un control "limitado", lo que genera atribuciones causales que podrían ser desencadenantes de la sintomatología ansiosodepresiva. Por el contrario, altas puntuaciones en Extraversión, que es el rasgo definitorio de las personas altamente sociables, que disfrutan compartiendo con otras personas y con tendencia a tener un estado de ánimo alegre, enérgico y optimista, se relaciona con una mayor disposición a atender las experiencias internas y externas, tales como sensaciones pensamientos o sentimientos y la capacidad de etiquetar con palabras la experiencia.

Del mismo modo, el factor Apertura a la experiencia, la integración activa, atención a los sentimientos interiores, preferencia por la variedad, la sensibilidad estética, la curiosidad intelectual y la independencia de juicio, se relaciona con la disposición a atender las experiencias externas e internas (sensaciones pensamientos o sentimientos), la capacidad de etiquetarlos y de permitir que discurran sin verse atrapado por ellos, es decir, con la faceta de No reaccionar.
Asimismo, el factor Responsabilidad se relaciona, sobre todo, con las facetas Describir y Actuar con conciencia, fundamentales para una buena autorregulación. Las personas que puntúan alto en responsabilidad presentan una gran capacidad de planificación y ejecución de las tareas, voluntariedad, decisión, motivación de logro. Todas estas cualidades están asociadas a un buen rendimiento académico o profesional. Es muy razonable pensar que cuanto mayor es la capacidad de la persona de centrarse en la actividad que está realizando y de etiquetar la experiencia interna, pueda tomar mejores decisiones que le lleven a un alto nivel de ejecución y un mayor rendimiento.

La Amabilidad, por el contrario, es el factor menos relacionado con la disposición al mindfulness; las personas amables y con tendencias interpersonales altruistas, empáticas, dispuestas a prestar ayuda y que son cooperadoras, también presentan una mayor disposición a no reaccionar a la experiencia y no juzgar, lo que podría favorecer el establecimiento de relaciones interpersonales positivas y saludables.

El segundo objetivo de este trabajo fue analizar las diferencias de sexo en la contribución de las facetas específicas de mindfulness a la sintomatología ansioso-depresiva, con la pretensión de analizar los mecanismos de actuación diferenciales de las facetas.

Con respecto a las diferencias de sexo en ansiedad se observa que, en el caso de las mujeres, las facetas de Describir, No juzgar y Actuar con conciencia pueden predecir las puntuaciones en síntomas de Ansiedad, Irritabilidad, Inutilidad y Rumiación. Por el contrario, en el caso de los hombres, las facetas con mayor capacidad predictiva de sintomatología ansiosa son No juzgar y No reaccionar. Posiblemente, para las mujeres con altas puntuaciones en Describir, que evalúa la capacidad de etiquetar con palabras la experiencia interna al prestar altos niveles de atención emocional, el hecho de etiquetar lo que sienten pueda producir un exceso de atención o una atención rumiativa, lo que a su vez generará más ansiedad. Debido a esto, las mujeres con mayor tendencia a juzgar esto es, a tener 
una actitud evaluativa hacia sus pensamientos y sentimientos, presentan altos niveles en problemas de pensamiento (Rumiación). Por el contrario, en el caso de los hombres con alta ansiedad, No reaccionar predice Depresión, Inutilidad y Rumiación, mientras que No juzgar predice Depresión, Irritabilidad y Rumiación.

Al estudiar a las diferencias de sexo en depresión, se encuentran diferencias llamativas según el género. En mujeres las facetas No juzgar y No reaccionar predicen Ansiedad, Depresión, Inutilidad, Irritabilidad y Rumiación. En hombres, ninguna faceta predice Ansiedad, pero No juzgar y No reaccionar predicen Depresión, Inutilidad, Irritabilidad y Rumiación. En el caso de los hombres, el mindfulness se relaciona más con depresión que con ansiedad. En el caso de las mujeres, habría que trabajar facetas (Describir, Observar y Actuar con conciencia en ansiedad, y sobre todo No juzgar) cuando hay síntomas depresivos. Todo esto nos indica que el tratamiento basado en mindfulness puede ser más útil en mujeres con altos niveles de ansiedad, mientras que puede ser más eficaz en el tratamiento de los hombres con alta ansiedad y depresión. Estos resultados van en la misma línea que otros trabajos que señalan la relación entre atención plena y salud mental, como por ejemplo los recogidos en la revisión realizada en 2017, por Tomlinson, Yousaf, Vitterso y Jones, cuya lectura recomendamos para una revisión más profunda.

Los resultados obtenidos apoyan la evidencia empírica existente de la función protectora de la faceta No juzgar sobre y más allá de otras facetas en lo que respecta a la depresión. Los hallazgos previos obtenidos sugieren que Rumiación media la relación existente entre la faceta No juzgar y la existencia de síntomas depresivos (Petrocchi \& Ottavianni, 2016). En el caso de los hombres con alta ansiedad y depresión, la faceta No juzgar parece ser la más relevante, seguida de No reaccionar.

Con respecto a la última hipótesis del estudio, solo encontramos diferencias significativas entre hombres y mujeres con altos niveles de Neuroticismo. Mientras que, en el caso de las mujeres, ocurre que cuanto menos atienden a sus experiencias internas y externas, tales como sensaciones pensamientos o sentimientos, se hallan menos niveles de Neuroticismo, en el hombre sucede que cuanto más observa, hay más Neuroticismo. Además, como se observa en la Tabla 4, es la tendencia a juzgar la que predice altos niveles de Neuroticismo, tanto en hombres como en mujeres. Una posible explicación puede ser que el juicio constante disminuya la sensación de control y los niveles de autoeficacia percibida.

La ausencia de mindfulness es lo que se denomina con el término mindlessness, que se caracteriza por la atención dirigida hacia el futuro o el pasado, la preocupación por los pensamientos fantasías y planes, y un comportamiento en piloto automático (Baer, Smith \& Allen, 2004). Es característica de las personas con alto nivel de neuroticismo. El entrenamiento en mindfulness podría actuar disminuyendo la tendencia a experimentar afecto negativo y facilitando el reappraisal cognitivo, el cual implica generar interpretaciones más positivas de las experiencias estresantes.

Entre las limitaciones del trabajo, podemos señalar el tamaño muestral, la deseabilidad social inherente a las medidas de autoinforme y la ausencia de población clínica con la que comparar los resultados obtenidos. En futuras investigaciones deberán tenerse en cuenta estos aspectos. Aunque las intervenciones basadas en mindfulness parecen moderadamente eficaces en la reducción de síntomas de depresión y ansiedad, deberán realizarse más estudios para establecer la eficacia de este tipo de tratamientos.

Los resultados de la presente investigación, a pesar de sus limitaciones, presentan implicaciones de cara a maximizar la eficacia las intervenciones basadas en mindfulness, ya que contribuyen a avanzar en la comprensión de los mecanismos de acción diferenciales entre hombres y mujeres, de manera que puedan ser empleados más eficazmente en contextos clínicos.

\section{Referencias}

Alonso, M. (2013). Mindfulness en el tratamiento de los trastornos de Ansiedad. 
En M. T. Miró y V. Simón (Eds.), Atención plena en la Práctica Clínica (pp. 81-141). Madrid: Desclee de Brouwe.

Aluja, A., Blanch, A., Solé, D., Dolcet J. M., \& Gallart, S. (2008). Validez convergente y estructural del neo-pi-r. Baremos orientativos. Boletín de Psicología, 92, 7-25.

Amutio-Kareaga, A., Franco Justo, C., Gázquez Linares, J. J, \& Mañas, I. (2015). Aprendizaje y práctica de la conciencia plena en estudiantes de bachillerato para potenciar la relajación y la autoeficacia en el rendimiento escolar. Universitas Psychologica, 14(2),433-443. https://www.d oi.org/10.11144/Javeriana.upsy14-2.apcp

Baer, R. A., Smith, G. T., \& Allen, K. B. (2004). Assessment of Mindfulness by self-report: The Kentucky inventory of Mindfulness skills. Assessment, 11 (3), 19-206. https://ww w.doi.org/10.1177/1073191104268029.

Baer, R. A., Smith, G. T., Hopkins, J., Krietemeyer, J., \& Toney, L. (2006). Using self- report assessment methods to explore facets of Mindfulness. Assessment, 13(1), 27-45. https://www.doi.org/10.1177/10731 91105283504

Bishop, S. R., Lau, M., Shapiro, S., Carlson, L., Anderson, N. D., Carmody, J., ...Devins, G. (2004). Mindfulness: A Proposed Operational Definition. Clinical Psychology: Science and Practice, 11 (3), 230-241. https:/ /www.doi.org/10.1093/clipsy.bph077

Bluth, K., Roberson, N., \& Girdler, S. (2017). Adolescent Sex Differences in Response to a Mindfulness Intervention: A Call for Research. Journal of Child and Family Studies, 26(7), 1900-1914.

Brown, K. W., \& Ryan, R. M. (2003). The benefits of being present: Mindfulness and its role in psychological well-being. Journal of Personality and Social Psychology, 84(4), 822-848. https://www.doi.org/10.1037/002 2-3514.84.4.822

Cebolla, A. (2013). Mindfulness en Terapia cognitiva para la depresión. En M. T. Miró \& V. Simón (Eds.), Mindfulness en la Práctica
Clínica (pp. 141-168). Bilbao: Desclée De Brouwer.

Cebolla, A., García-Palacios, A., Soler, J., Guillen, V., Baños, R., \& Botella, C. (2012). Psychometric properties of the Spanish validation of the Five Facets of Mindfulness Questionnaire (FFMQ). The European Journal of Psychiatry, 26(2), 118-126. https://doi.org/10.4321/S0213-61 632012000200005

Ciesla, J. A., Reilly, L. C., Dickson, K. S., Emanuel, A. S., Updegraff, J. A. (2012). Dispositional Mindfulness moderates the effects of stress among adolescents: rumination as a mediator. Journal of Clinical Child Adolescent Psychology, 41(6), 760-770. https://doi.org/10.1080/15374416 .2012 .698724

Cuevas-Toro, A. M., Díaz-Batanero, C., Delgado-Rico, E., \& Vélez-Toral, M. (2017). Incorporación del mindfulness en el aula: un estudio piloto con estudiantes universitarios. Universitas Psychologica, 16(4), 54-66. https://www.doi.org/10.1114 4/javeriana.upsy16-4.imae

Desrosiers, A., Klemanski, D. H., \& Nolen-Hoeksema, H. (2013). Mapping Mindfulness facets onto dimensions of anxiety and depression. Behavior Therapy, 44(3), 373-84.

Dixon, H., \& Overal, N. (2016). Dispositional Mindfulness Attenuates the link Between Daily Stress and Depressed Mood. Journal of Social and Clinical Psychology, 35(3), 255-268.

España, Ministerio de Sanidad, Consumo y Bienestar Social. (2019). Encuesta Nacional de Salud ENSE, España de 2017. Serie informes monográficos \#1. Salud Mental. Madrid: Autor.

Feagans, L., Dariotis J., Mendelson, T., \& Greenberg, M. (2012). A school-based Mindfulness intervention for urban youth: exploring moderators of intervention effects. Journal of Community Psychology, 40 (8), 968-982. https://doi.org/10.1002/jco p.21505 
Geiger, P. J., Boggero, I. A., Brake, C. A., Caldera, C. A., Combs, H. L., Peters, J. R., \& Baer, R. A. (2016). Mindfulness-Based Interventions for Older Adults: a Review of the Effects on Physical and Emotional WellBeing. Mindfulness, 7(2), 296-307. https://d oi.org/10.1007/s12671-015-0444-1

Goyal, M., Singh, S., Sibinga, E. M. S., Gould, N. F., Rowland-Seymour, A., Sharma, R., ... Haythornthwaite, J. A. (2014). Meditation programs for psychological stress and wellbeing: a systematic review and metaanalisis. JAMA International Medicine, 174(3), 357-368. https://doi.org/10.1001/ja mainternmed.2013.13018

Greeson, J. M., Smoski, M. J., Suarez, E. C., Brantley, J. G., Ekblad, A. G., Lynch, T. R., \& Wolever, R. Q. (2015). Decreased Symptoms of Depression After Mindfulness-Based Stress Reduction: Potential Moderating Effects of Religiosity, Spirituality, Trait Mindfulness, Sex, and Age. The Journal of Alternative and Complementary Medicine, 21(3), 166-174. h ttps://doi.org/10.1089/acm.2014.0285

Hassed, C., de Lisle, S., Sullivan, G., \& Pier, C. (2009). Enhancing the health of medical students: outcomes of an integrated mindfulness and lifestyle program. Advances in Health Sciences Education, 14(3), 387-398. https://doi.org/1 0.1007/s10459-008-9125-3

Hervás, G., Cebolla, A., \& Soler, J. (2016) Intervenciones psicológicas basadas en Mindfulness y sus beneficios: estado actual de la cuestión. Clínica y Salud, 27(3), 115-124.

Hoffman, S. G., Sawyer, A. T., \& Witt, A. (2010). The effect of Mindfulness-based therapy on anxiety and depression: a metaanalitic review. Journal Consult Clinical Psychological, 78(2), 169-183. https://doi.or $\mathrm{g} / 10.1037 / \mathrm{a} 0018555$

Jankowski, T., \& Holas, P. (2014). Metacognitive model of Mindfulness. Consciousness and Cognition, 28, 64-80. https://doi.org/10.101 6/j.concog.2014.06.005
Justo, C., de la Fuente, M., \& Salvador, M. (2011). Impacto de un programa de entrenamiento en conciencia plena en las medidas de crecimiento y la autorrealización personal. Psicothema, 23(1), 58-65.

Kabat-Zinn, J. (2003). Mindfulness-Based Interventions in Context: Past, Present, and Future. Clinical Psychology: Science and Practice, 10(2), 144-156. https://doi.org/10. 1093/clipsy/bpg016

Khoury, B., Lecomte, T., Fortin, G., Masse, M., Therien, P., Bouchard, V., ...Hofmann, S. G. (2013). Mindfulness based therapy: a comprehensive meta-analysis. Clinical Psychological Review, 33(6), 763-771.

Lang, A. J. (2013), What Mindfulness brings to psychotherapy for anxiety and depression. Depression and Anxiety, 30(5), 409-412. htt ps://doi.org/10.1002/da.22081

Lozano, L., García-Cueto, E., \& Lozano, L. M. (2007). Cecad. Cuestionario EducativoClínico: ansiedad y depresión. Madrid: TEA Ediciones.

Manee, M., \& Majreh, A. (2016). The correlation of neuroticism with symptoms of depression and anxiety in students according to the mediating role of worry and rumination. Journal of Research $\mathbb{E}$ Health, 6(1), 175-185.

Mathews, A., \& MacLeod, C. (2005). Cognitive Vulnerability to Emotional Disorders. Annual Review of Clinical Psychology, 1, 167-195. https://doi.org/10.1146/annurev.c linpsy.1.102803.143916

Metcalf, C., Gold, A., Davis, B., Sylvia, L., \& Battle, C. (2019). Mindfulness as an Intervention for Depression. Psychiatric Annals, 49(1), 16-20. https://doi.org/10.39 28/00485713-20181205-01

Pascual, A., Conejero, S., \& Etxebarria, I. (2016). Coping strategies and emotion regulation in adolescents: Adequacy and gender difference. Ansiedad y Estrés, 22(1), 1-4. https://doi.org/10.1016/j.anyes.2016.0 4.002

Petrocchi, N., \& Ottavianni, C. (2016). Mindfulness facets distinctively predict 
depressive symptoms after two years: The mediating role of rumination. Personality $\mathfrak{E}$ Individual Differences, 93, 92-96.

Raphiphatthana, B., Jose, P. E., \& Kielpikowski, M. (2016). How do the facets of Mindfulness predict the constructs of depression and anxiety as seen through the lens of the tripartite theory? Personality $\mathbb{E}$ Individual Differences, 93, 104-111. https://d oi.org/10.1016/j.paid.2015.08.005

Sanz, J., Silva, F., \& Avia, M. D. (1999). La evaluación de la personalidad desde el modelo de los "Cinco Grandes": El Inventario de Cinco Factores NEO de Costa y McCrae. En F. Silva (Ed.), Avances en Evaluación Psicológica (pp. 171-234). Valencia: Promolibro.

Smidt, C., \& Vinet, E. (2015). Atención Plena: Validación del Five Facet Mindfulness Questionarire en estudiantes universitarios Chilenos. Terapia Psicológica, 33(2), 93-102.

Strauss, C., Cavanag, K., Oliver, A., \& Pettman, D. (2014). Mindfulness-Based Interventions for people Diagnosed with a Current Episode of an Anxiety or Depressive Disorder: A meta-analysis of randomized controlled trials. PLoS ONE, 9(4), 1-13. https://doi.org/10.1371/journal. pone.0096110

Tomlinson, E., Yousaf, O., Vitterso, A., \& Jones, L. (2017). Dispositional Mindfulnnes and Psychological Health: a systematic Review. Mindfulness, 9(1), 1-21. https://doi.org/10.1 007/s12671-017-0762-6

Wazczuk, M., Zavos, H., Antonova, E., Haworth, C., Plomin, R., \& Eley, T. (2015). A Multivariate twin study of trait Mindfulness, depressive symptoms, and anxiety sensitivity. Depression and Anxiety, 32 (4), 254-261. https://doi.org/10.1002/da. 22326

Wharton, E., \& Kanas, N. (2019). MindfulnessBased Stress Reduction for the Treatment of Anxiety Disorders. International Journal of Group Psychotherapy, 69(3), 1-11. https:/ /doi.org/10.1080/00207284.2019.1599289.

Zeng, W., \& Gu, M. (2017). Relationship between Mindfulness and positive affect of Chinese older adults: optimism as mediator. Social Behavior and Personality, 45(1), 155-162.

Notas

* Artículo de investigación. 